\title{
Resistance to the tyrosine kinase inhibitor axitinib is associated with increased glucose metabolism in pancreatic adenocarcinoma
}

\author{
CD Hudson ${ }^{1,2}$, T Hagemann ${ }^{2}$, SJ Mather ${ }^{2}$ and N Avril ${ }^{\star, 1,2}$
}

Alterations in energy (glucose) metabolism are key events in the development and progression of cancer. In pancreatic adenocarcinoma (PDAC) cells, we investigated changes in glucose metabolism induced by resistance to the receptor tyrosine kinase inhibitor (RTKI) axitinib. Here, we show that human cell lines and mouse PDAC cell lines obtained from the spontaneous pancreatic cancer mouse model (Kras ${ }^{\mathrm{G} 12 \mathrm{D}} \mathrm{Pdx1}$-cre) were sensitive to axitinib. The anti-proliferative effect was due to a G2/M block resulting in loss of 70-75\% cell viability in the most sensitive PDAC cell line. However, a surviving sub-population showed a 2- to 3-fold increase in [C-14]deoxyglucose ([C-14]DG) uptake. This was sustained in axitinib-resistant cell lines, which were derived from parental PDAC. In addition to the axitinib-induced increase in [C-14]DG uptake, we observed a translocation of glucose transporter-1 (Glut-1) transporters from cytosolic pools to the cell surface membrane and a 2-fold increase in glycolysis rates measured by the extracellular acidification rate (ECAR). We demonstrated an axitinib-induced increase in phosphorylated Protein Kinase B (pAkt) and by blocking pAkt with a phosphatidylinositol-3 kinase (PI3K) inhibitor we reversed the Glut-1 translocation and restored sensitivity to axitinib treatment. Combination treatment with both axitinib and Akt inhibitor in parental pancreatic cell line resulted in a decrease in cell viability beyond that conferred by single therapy alone. Our study shows that PDAC resistance to axitinib results in increased glucose metabolism mediated by activated Akt. Combining axitinib and an Akt inhibitor may improve treatment in PDAC.

Cell Death and Disease (2014) 5, e1160; doi:10.1038/cddis.2014.125; published online 10 April 2014

Subject Category: Cancer Metabolism

Despite multimodality treatment, metastatic pancreatic adenocarcinoma (PDAC) has a 5-year survival rate of only $3-5 \%{ }^{1}$ Early stages of the disease are treated with aggressive surgery; however, due to the often advanced stage of disease at initial presentation the front-line treatment is chemotherapy. Gemcitabine, a nucleoside analog inhibiting DNA synthesis, is the most often used chemotherapeutic agent for treatment of PDAC. Tyrosine kinase inhibitors (TKI) are anti-cancer drugs used to inhibit the aberrant activated tyrosine kinase signaling pathways in cancer cells. ${ }^{2}$ The high levels of vascular endothelial growth factor (VEGF) in pancreatic cancer suggest that combination therapies which include an antiangiogenic TKI may improve treatment. Axitinib inhibits VEGF receptor (VEGFR)-1, -2 and -3 and to a lesser extent plateletderived growth factor receptors (PDGFRs) and C-Kit. ${ }^{3}$ All of these targets are known to be deregulated and overexpressed in pancreatic cancer. ${ }^{4-6}$ In phase II clinical trials, axitinib has shown anti-tumor effects in a range of cancers including metastatic renal cancer, ${ }^{7,8}$ pancreatic cancer, ${ }^{9}$ thyroid cancer, ${ }^{10}$ NSCLC ${ }^{11}$ and melanoma. ${ }^{12}$ A phase II clinical trial in patients with advanced PDAC showed improved survival in combination with gemcitabine, ${ }^{9}$ however, a subsequent phase III clinical trial did not confirm improved survival rates. ${ }^{13}$ Resistance to TKI treatment is complex and includes increased drug efflux, drug inactivation, enhanced DNA damage repair and activation of pro-survival signaling. ${ }^{14}$ More recently, increased tumor glucose metabolism has been linked to resistance to $\mathrm{TKI}$ treatment. ${ }^{15} \mathrm{We}$ therefore investigated changes in activated Protein Kinase B (Akt) and glucose metabolism during axitinib treatment and in axitinib-resistant cell lines.

We used human cell lines and mouse PDAC cell lines obtained from the spontaneous pancreatic cancer mouse model (Kras $\left.{ }^{G 12 D} P d x 1-c r e\right)$, which recapitulates the features of human PDAC. The translational aspect of our research is emphasized by many types of cancers that are being characterized by an increase in glucose metabolism, which can be visualized by positron emission tomography (PET)

\footnotetext{
${ }^{1}$ Case Western Reserve University, University Hospitals Case Medical Center, Cleveland, OH, USA and ${ }^{2}$ Centre for Molecular Oncology, Barts Cancer Institute, Barts and The London School of Medicine and Dentistry, Queen Mary University of London, London, UK

*Corresponding author: N Avril, Case Western Reserve University, University Hospitals Case Medical Center, 11100 Euclid Avenue, BSH 5056, Cleveland, OH 44106, USA. Tel: +1 216286 3210; Fax: +1 216286 3209; E-mail: Norbert.Avril@Case.edu

Keywords: receptor tyrosine kinase inhibitor; pancreatic adenocarcinoma; PI3K/Akt; glycolysis; drug resistance; molecular targeted therapies

Abbreviations: Akt, Protein Kinase B; [C-14]DG, [C-14]deoxyglucose; DAPI, 4,6-diamidino-2-phenylindole; ECAR, extracellular acidification rate; EGFR, epidermal growth factor receptor; FDG, Fluorine-18 labeled [F18] fluorodeoxyglucose; FITC, fluorescein isothiocyanate; Glut-1, glucose transporter-1; MCT-4, monocarboxylate transporter 4; mTORC1, mammalian target of rapamycin complex 1; PDAC, pancreatic adenocarcinoma; PDGFR, platelet-derived growth factor receptor; PET, positron emission tomography; PI, propidium iodide; PI3K, phosphatidylinositol-3 kinase; RTKI, receptor tyrosine kinase inhibitor; VEGFR, vascular endothelial growth factor receptor

Received 10.9.13; revised 14.2.14; accepted 18.2.14; Edited by C Munoz-Pinedo
} 
using Fluorine-18 labeled [F18] fluorodeoxyglucose (FDG). ${ }^{16-22}$ FDG-PET has gained a significant clinical importance for diagnosis and treatment monitoring of cancer patients over the past decade. Tumor glucose metabolism provides important information about cell viability and early treatment effects and FDG-PET has the potential to serve as a surrogate marker of treatment response to new targeted treatments.

\section{Results}

Axitinib treatment inhibits pancreatic cell proliferation and induces G2/M cell-cycle arrest but prolonged treatment leads to drug resistance. Axitinib reduced cell viability in a dose-dependent manner and after $24 \mathrm{~h}$ exposure, the $\mathrm{IC}_{50}$ doses were $0.44,0.69,0.74$ and $0.82 \mu \mathrm{M}$ for mouse PDAC and human pancreatic cancer cell lines Miapaca, BXPC-3 and Panc1, respectively (Figure 1a). After $48 \mathrm{~h}$ exposure, the $\mathrm{IC}_{50}$ doses for each cell line were not significantly further decreased. Although the mouse PDAC cells showed the greatest sensitivity to axitinib, there was a remaining $25-30 \%$ population of cells that survived even when treated with high doses of axitinib and at longer incubation time points (data not shown). These remaining surviving cells showed a distinct morphology and were up to 10 times bigger than untreated cells (Figures $1 b$ and $c$ ).

To investigate the effects of axitinib on PDAC cell cycle, we focused on the mouse PDAC cell line that exhibited the highest sensitivity to axitinib. Cell-cycle analysis showed that increasing concentrations of axitinib led to an increase in the percentage of cells in G2/M cell-cycle arrest with a concomitant decrease in cells in G0/G1 after $24 \mathrm{~h}$, which was statistically significant at doses of $0.25 \mu \mathrm{M}$ and higher $(P<0.05$, Figure 1d). These results show that the antiproliferative effect of axitinib is mediated via a cell-cycle arrest at the G2/M checkpoint rather than a direct increase in apoptosis.

The persistence of a sub-population that survived even using longer incubation times or higher concentrations of axitinib implies the selection of resistant cells. To investigate the relationship between repeated axitinib treatment and drug resistance, we pulse treated mouse PDAC cells with $0.5 \mu \mathrm{M}$ axitinib for $48 \mathrm{~h}$ for eight cycles, allowing the cells to recover between cycles. Subsequently, three resistant clones were isolated from single cells. Viability assays showed that whereas the mouse PDAC parental cell line was highly sensitive to axitinib, the resistant PDAC clones (mouse PDAC-R1, PDAC-R2 and PDAC-R3) were not growth inhibited even at $1 \mu \mathrm{M}$ concentration of axitinib (Figure 1e). Furthermore, the resistant cell lines failed to show the G2/M arrest in response to increasing concentrations of axitinib (Figure 1f) that was seen in the parental cell line (Figure 1d).

\footnotetext{
Axitinib treatment increases glucose uptake in the remaining axitinib surviving population. Since major oncogenic signaling pathways have been linked to increased glucose metabolism, we hypothesized that blocking them with a TKI would decrease glucose uptake. However, we found that [C-14]deoxyglucose ([C-14]DG) uptake was increased in axitinib-treated cells after $24 \mathrm{~h}$ (Figure 2a) and
}

$48 \mathrm{~h}$ treatment (Figure $2 \mathrm{~b}$ ). This effect was greatest in the mouse PDAC cell line. At $0.25,0.5,0.75$ and $1 \mu \mathrm{M}$ there was a 162, 230, 270 and 327\% increase in [C-14]DG uptake after $24 \mathrm{~h}$ (nmole uptake per 10000 viable cells, expressed relative to untreated control). This was maintained in the axitinib-resistant PDAC-R1, -R2 and -R3 clones, all of which show a 2-fold increase in [C-14]DG uptake compared with the parental PDAC cell line (Figure 2c). Furthermore, no further additional increase in [C-14]DG uptake was observed in the axitinib-resistant cell lines treated with 0.5 or $1 \mu \mathrm{M}$ axitinib for $24 \mathrm{~h}$ compared with the untreated resistant cell lines (Figure 2c). This suggests that the increased glucose radiotracer uptake is involved in the resistance mechanism toward axitinib-induced anti-tumor effects. We next sought to determine whether the axitinib-resistant cell lines were more sensitive to growth inhibition and /or cell death induced by glucose deprivation or treatment with 2-DG, a glucose analog that cannot be metabolized by the cells. The PDAC-resistant clones were more sensitive to glucose deprivation compared with the parental PDAC cell line after culture in glucose-free media over $48 \mathrm{~h}$ (Figure $2 \mathrm{~d}$ ). Similarly, the resistant cell lines were more sensitive to $2-D G$ treatment showing a $45 \%$ decrease in cell viability compared with $20 \%$ in the parental cell line (Figure 2d).

Axitinib results in increased glucose transporter-1 cell surface expression and glycolysis. To investigate the molecular basis of increased [C-14]DG uptake in response to axitinib treatment, we examined the surface expression of glucose transporter-1 (Glut-1) protein by FACS and mRNA using reverse transcriptase-PCR (RT-PCR). Analysis of Glut-1 surface expression by flow cytometry revealed that treatment of PDAC cells with increasing concentrations of axitinib upregulated Glut-1 surface expression in a dosedependent manner (Figure 3a). However, analysis of Glut-1 mRNA by RT-PCR in PDAC cells treated with increasing concentrations of axitinib led to only a modest increase (Figure $3 b$ ). These results suggest that the axitinib-induced increased Glut-1 surface expression is most likely regulated at the posttranslational level. To assess protein stabilization, western blots were carried out on PDAC cells treated with $1 \mu \mathrm{m}$ axitinib for $10 \mathrm{~min}$ to $24 \mathrm{~h}$. However, no significant overall increase in Glut-1 protein in response to axitinib treatment was seen (Figure 3c). We therefore investigated whether the increased cell surface expression of Glut-1 might be due to translocation from intracellular cytoplasmic pools to the cell membrane. Glut-1 localization experiments using confocal fluorescence microscopy showed that Glut-1 localization in untreated cells is concentrated in cytoplasmic pools (Figure 3d). However, upon axitinib treatment, this localization is more dispersed throughout the cytoplasm with the formation of rings around the cellular membrane (Figure 3e). Therefore, we conclude that axitinib resistance in PDAC cells induces increased glucose uptake with Glut-1 translocation from intracellular cytoplasmic pools to the cell membrane.

Measurement of the extracellular acidification rate (ECAR), which reflects glycolytic rates, was also measured in mouse PDAC cells in response to axitinib treatment. Cells treated with axitinib for $24 \mathrm{~h}$ that showed increased Glut- 1 cell surface 
a

Pancreatic cell survival
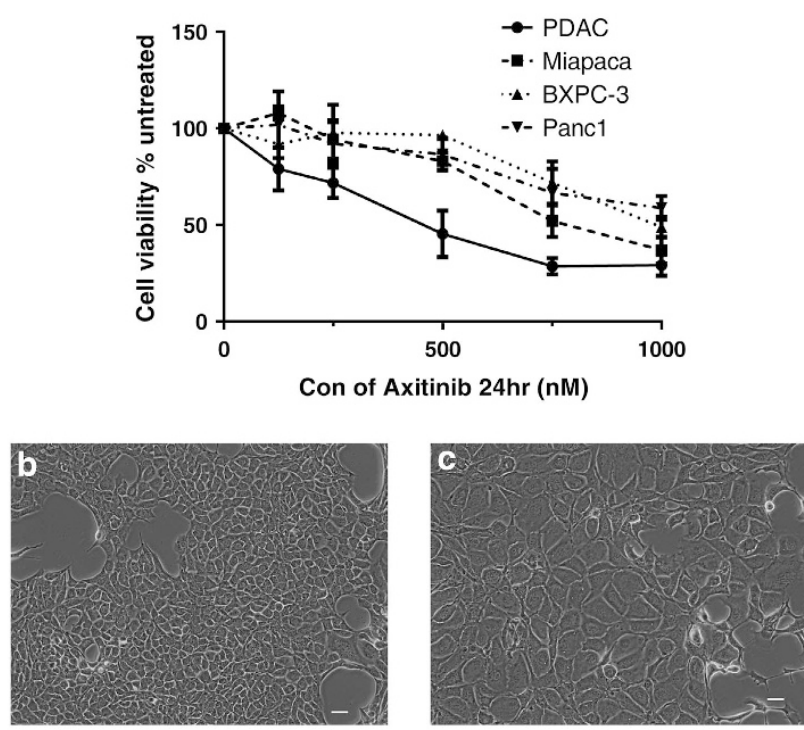

d

Mouse PDAC cells

e
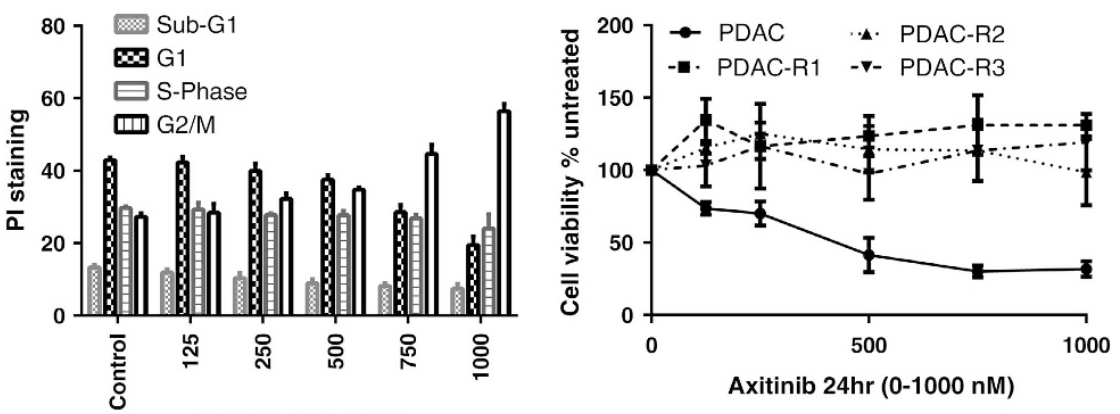

Axitinib 24hr (nM)

f

PDAC-R1

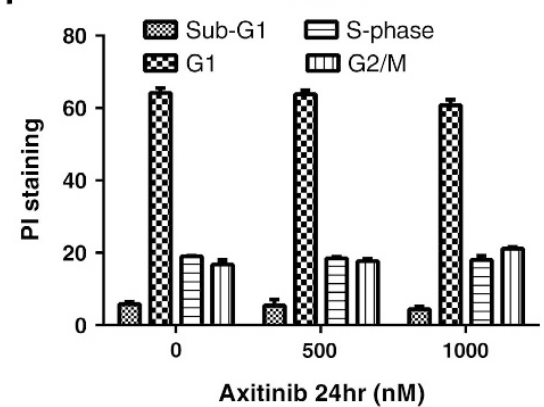

Figure 1 Axitinib inhibits pancreatic cell growth, causes a G2/M cell-cycle arrest and affects cell morphology. (a) Sensitivity of pancreatic cell lines to axitinib (0.125-1 $\mu \mathrm{M}$ ) for $24 \mathrm{~h}$ was assessed by Vi-CELL trypan blue counter. Cell viability was calculated as the ratio of treated to untreated cells at each dose for each cell line. Experiments were performed in duplicate and repeated three times. Data presented as mean \pm S.D. $(n=6)$. (b and $\mathbf{c})$ Mouse PDAC cells were photographed before and after treatment with $1 \mu \mathrm{m}$ axitinib. Cells are reduced in number and are $\times 10$ bigger after treatment. Scale bar, $50 \mu \mathrm{m}$. (d) Cells were treated with DMSO or with Axitinib $(0.125-1 \mu \mathrm{m})$ for $24 \mathrm{~h}$, stained with propidium iodide and analyzed for cell-cycle progression by flow cytometry. Treatment resulted in an increased G2/M block $(P<0.05)$, and data represent mean \pm S.D. of three experiments performed in triplicate $(n=9)$. (e) Sensitivity of mouse PDAC and mouse axitinib-resistant clones PDAC-R1, R2 and R3 to axitinib treatment for $24 \mathrm{~h}$. Experiments were performed in duplicate and repeated three times. Data presented as mean \pm S.D. $(n=6)$. ( $f$ ) Resistant cell lines PDAC-R1, R2 and R3 were treated with DMSO or $1 \mu \mathrm{M}$ axitinib for $24 \mathrm{~h}$. Cells were harvested, fixed and stained with propidium iodide and analyzed by flow cytometry. Data represent one cell clone

expression compared with untreated, and increased glucose uptake also displayed a 2-fold increase in glycolysis rates when treated with axitinib concentration at or above $500 \mathrm{nM}$ (Figure 4a). Furthermore, the increased glycolytic rate (2-fold) is maintained in the PDAC-R1, R2- and R3-resistant cell lines and is not significantly altered by the addition of $1 \mu \mathrm{M}$ axitinib for $24 \mathrm{~h}$ (Figure $4 \mathrm{a}$-showing results for mouse PDAC-R1 that is representative of the three clones). To confirm the 
a

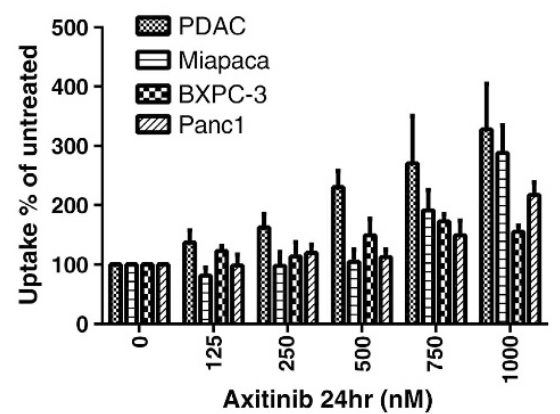

C

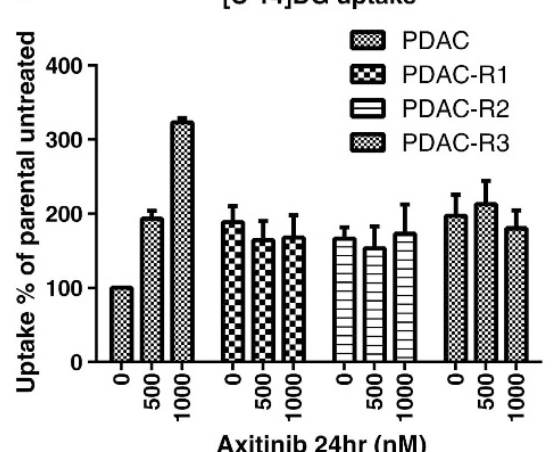

b

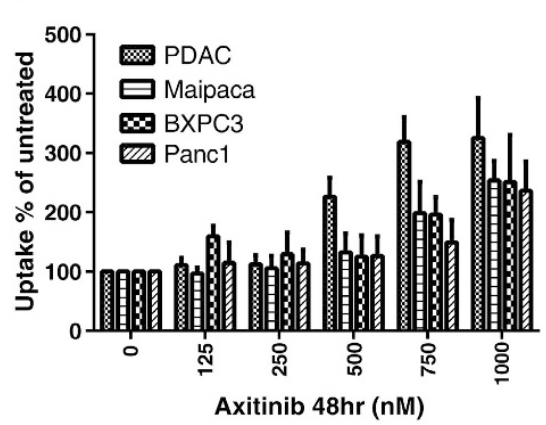

d

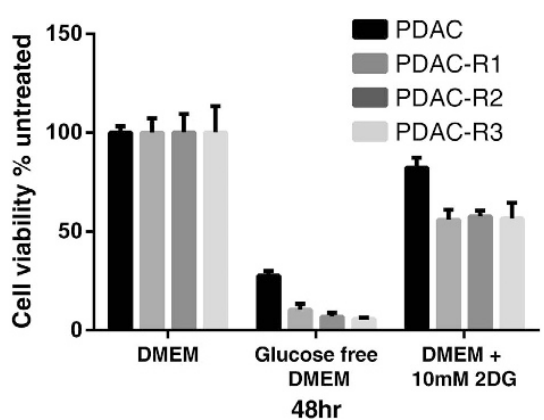

Figure 2 Axitinib increases glucose uptake in the remaining surviving population. ( $\mathbf{a}$ and $\mathbf{b})$ [C14]-2DG uptake in pancreatic cells after treatment with axitinib $(0.125-1 \mu \mathrm{M})$. Uptake is normalized to cell number. The cells remaining after axitinib treatment increase their glucose uptake per 10000 cells with increasing concentrations of axitinib after 24 and $48 \mathrm{~h}$ treatment $(P<0.05)$. (c) The resistant mouse PDAC cell lines display a 2 -fold increased glucose uptake compared with the parental cell line $(P<0.05)$. No further increase in tracer uptake is found when the resistant cell lines are treated with axitinib. (d) Parental PDAC and mouse PDAC-resistant cell lines were cultured in complete media, glucose free or complete media containing 2-DG $(10 \mathrm{mM})$. Cell viability was determined using the Vi-CELL trypan blue exclusion counter. Experiments were performed in triplicate and repeated three times and data are expressed relative to untreated control or parental cell line. Data represent mean and S.D. $(n=9)$

increased lactate efflux as a result of increased glycolysis, we also assessed the expression of the monocarboxylate transporter 4 (MCT-4) which is highly expressed in tumor cells to mediate lactic acid efflux. Western blot analysis showed increased MCT-4 protein in the mouse PDAC cells after axitinib treatment and also in the axitinib-resistant cells compared with untreated parental PDAC cells $(P<0.01$, Figure 4b).

Increased Glut-1 cell surface expression in cells surviving axitinib treatment is mediated by increased activation of Akt. Akt has a key role in multiple cellular processes such as glucose metabolism and cell proliferation and survival. We therefore investigated the effect of axitinib on phosphorylated Akt (pAkt) levels. Though basal levels of pAkt were seen in untreated mouse PDAC cells, treatment with $1 \mu \mathrm{M}$ axitinib resulted in increased pAkt as early as $30 \mathrm{~min}$, which gradually declined after $4 \mathrm{~h}$ but remained at higher levels compared with untreated PDAC $(P<0.05)$. After $24 \mathrm{~h}$ treatment with $1 \mu \mathrm{M}$ axitinib, higher pAkt levels were sustained compared with untreated control $(P<0.05)$ (Figures $5 a$ and b).

To investigate whether the increased surface expression of Glut-1 is mediated via axitinib-induced increased pAkt levels, we evaluated the surface expression of Glut-1 in the presence of axitinib and the phosphatidylinositol-3 kinase (PI3K)/Akt inhibitor LY294002. In all, $1 \mu \mathrm{M}$ of LY294002 alone reduced the level of Glut-1 surface expression to below that seen in untreated cells and LY294002 also reversed the increase in surface Glut-1 expression induced by either 0.5 or $1 \mu \mathrm{M}$ axitinib treatment (Figure $5 \mathrm{c}$ ). Western blot confirmed the decreased levels of pAkt in the combinations of drugs that resulted in decreased Glut-1 surface expression (Figure $5 d$ ). Together, these results demonstrated that pAkt mediates the increased Glut-1 surface expression we observed upon axitinib treatment.

Akt-specific inhibitor sensitizes PDAC-resistant clones to axitinib and decreases cell viability in PDAC cells. We investigated whether the Akt-specific inhibitor MK-2206 sensitizes axitinib-resistant mouse PDAC cells to the re-exposure of axitinib treatment. As illustrated in Figure $6 \mathrm{a}$, the number of viable cells of PDAC-R1, the axitinib-resistant mouse PDAC cell line, was unaffected by MK-2206 alone (range $0-30 \mu \mathrm{M}, 24 \mathrm{~h}$ treatment). However, increasing doses of MK-2206 sensitized the previously axitinib-resistant cells to axitinib treatment (Figure 6a). The effect of a combined Akt inhibitor/axitinib treatment was further tested in PDAC cell lines. We found that combined treatment with increasing concentrations of MK-2206 together with axitinib at the $I C_{10}$ concentration ( $24 \mathrm{~h}$ treatment) led to a further $45 \%$ decrease in cell viability compared with MK-2206 alone and a further 

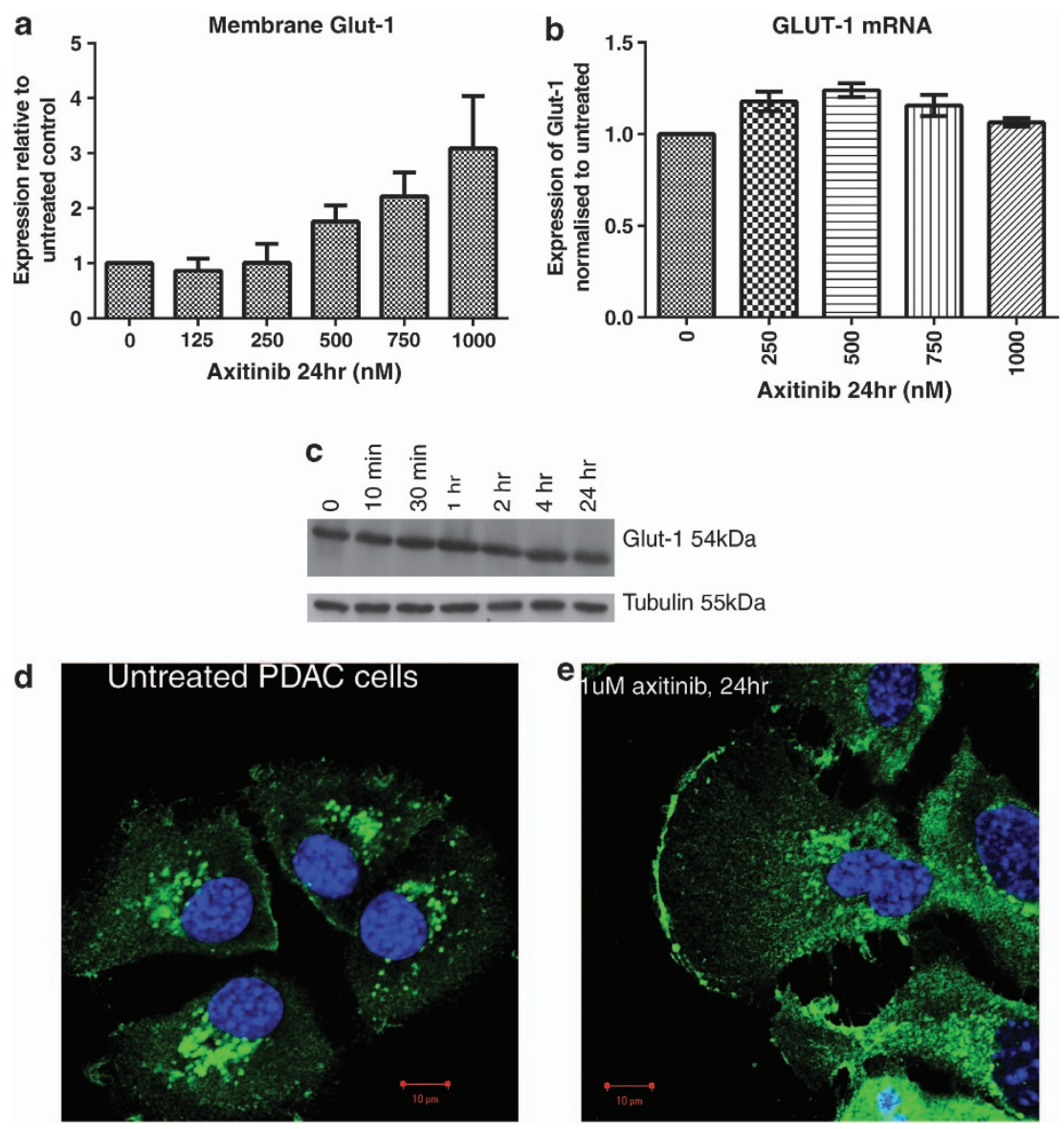

Figure 3 Axitinib triggers enhanced Glut-1 cell surface expression by increasing translocation from intracellular pools to the membrane. (a) Glut-1 surface expression was quantified by flow cytometry using Glut-1 conjugated to PE. Graph represents increased Glut-1 expression upon increasing concentrations of axitinib relative to untreated control. Experiments were performed in duplicate and repeated at least three times. Data are mean and S.D. $(n=+6)$. (b) RT-PCR was used to assess expression level of Glut-1 in cells treated with axitinib $(0.250-1 \mu \mathrm{M})$ compared with untreated controls. (c) Western blot showing levels of Glut-1 protein in response to axitinib treatment. (d and $\mathbf{e})$ Cellular localization of Glut-1 protein by confocal immunofluorescent staining. PDAC cells were probed with anti-Glut-1 (Millipore) at a dilution of $1 / 100$ for $1 \mathrm{~h}$. Localization of bound antibodies was visualized by Alexa Fluor 488 conjugated secondary antibody (Green), at a dilution of 1/2000 for 30 min. The cell nucleus is stained with DAPI (blue). (d) Glut-1 (green) is concentrated in cytoplasmic pools in the untreated PDAC cells. (e) After axitinib treatment Glut-1 translocates and accumulates at the cytoplasmic membrane from the intracellular pools. Scale bar, $10 \mu \mathrm{m}$

$55 \%$ decrease in cell viability compared with axitinib alone $\left(\mathrm{IC}_{10}\right)$ (Figure $6 \mathrm{~b}$ ). Inhibition of Akt-mediated glucose transport therefore restores axitinib-mediated cell death and dual treatment with MK-2206 and axitinib shows promise for future investigation in the treatment of PDAC.

\section{Discussion}

We demonstrated a direct toxic effect of axitinib on mouse and human PDAC cell lines with an $\mathrm{IC}_{50}$ ranging from 0.44 to $0.82 \mu \mathrm{M}$, which is comparable to the previously published data. $^{3,23,24}$ Axitinib caused a significant reduction in cell proliferation in PDAC cells resulting from a G2/M block (Figure 1d). Canu et al. ${ }^{24}$ previously found that axitinib directly inhibits the proliferation as well as increasing the apoptotic rate in the pancreatic cell lines Capan-1 and Miapaca.

We used [C-14]DG uptake assays to assess treatmentinduced changes in glucose metabolism and found a 2- to 4-fold increase in glucose metabolism in surviving viable PDAC cells (Figure 2). This effect was also observed for the chemotherapeutic agent gemcitabine (data not shown). Although the molecular mechanism of an early increase in glucose metabolism following treatment is not yet fully understood, it is in line with a report from Haberkorn et al. ${ }^{25}$ who found enhanced uptake of FDG and 3-O-methylglucose in rat prostate adenocarcinoma cells in vitro and a significant increase in glucose transport rates and phosphorylation in vivo after $24 \mathrm{~h}$ of treatment with gemcitabine.

Subsequently, stable resistant mouse PDAC cell clones were isolated following pulse treatment with axitinib over a 6-month period. Of note, these cell lines also showed a 2-fold increase in [C-14]DG uptake compared with the parental cell line, suggesting an important role of glucose metabolism in the development of resistance. Furthermore, the axitinibresistant PDAC cell lines were more sensitive to glucose deprivation and treatment with the glucose analog, 2-DG compared with the parental cell line as shown in Figure $2 \mathrm{~d}$. To confirm the observed increased glucose metabolism, we performed ECAR assays using XF24 analyzer (Seahorse Biosciences, Boston, MA, USA), which measures in real time 


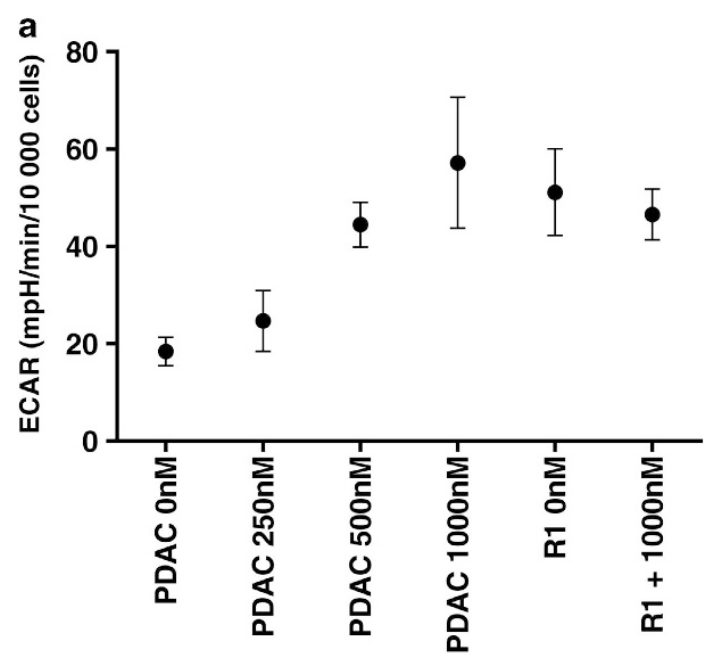

Axitinib 24hr

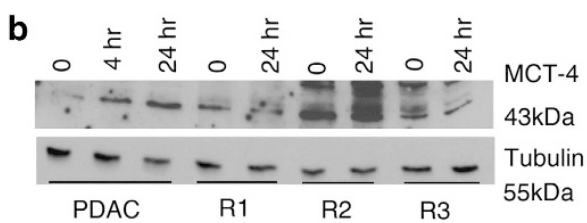

Figure 4 Axitinib treatment increases glycolysis. (a) Mouse PDAC and axitinibresistant mouse PDAC cell lines were treated with DMSO or with increasing concentrations of axitinib for $24 \mathrm{~h}$ before XF measurement. Glycolysis is increased in treated mouse PDAC cells and resistant cell lines compared with untreated parental control. Exposure of resistant cell lines to $1 \mu \mathrm{M}$ axitinib does not significantly alter EACR rates. Mouse PDAC-R1 is shown as representative of the resistant cell lines. Experiments were carried out in triplicates and repeated three times. Data represent mean and S.D. $(n=9)$. (b) Western blot showing increased MCT-4 expression in treated PDAC cells and in the resistant cell lines compared with untreated PDAC control

the uptake and release of metabolic end products. We found a dose-dependent increase in glycolytic rates in the surviving cell population following $24 \mathrm{~h}$ of axitinib treatment in the parental PDAC cell line (Figure 4a). Furthermore, the glycolytic rate in the axitinib-resistant PDAC cell lines was 2-fold higher compared with untreated parental control and treatment of the resistant cell lines with axitinib resulted in no further changes in glycolytic rates (Figure 4a). However, lactate release is not the only cause of medium acidification. Lactate is transported out of the cell via the MCTs. MCT-4 is mostly associated with the export of lactate in cells with high glycolytic rates, ${ }^{26}$ and is highly expressed in pancreatic cancer. ${ }^{27}$ We confirm the increased glycolysis is matched by increased levels of MCT-4 protein in the axitinib-treated PDAC cells and the axitinib-resistant PDAC-R1, $-2,-3$ clones, compared with untreated PDAC cells.

The transport of glucose through the cell membrane is mediated by glucose transport proteins, and Glut- 1 is often overexpressed in cancer cells and correlates with decreased response to therapy. ${ }^{28-30}$ Flow cytometry and confocal microscopy showed an increased cell surface expression of Glut-1 in response to axitinib treatment. This was not caused by a de novo synthesis of Glut-1 protein but by a translocation of Glut-1 from cytosolic pools to the cell surface membrane
(Figures $3 d$ and e). No changes in Glut-1 mRNA confirmed the post translational regulation. A translocation of glucose transport proteins has previously been reported following the treatment of GIST tumors with the TKI imatinib, which resulted in decreased Glut-1 cell surface expression. ${ }^{31}$ Within hours of treatment the increased glucose metabolism is significantly reduced in such tumors, which can be seen clinically using FDG-PET.

Although the mechanisms remain unclear, resistance to targeted treatment of cell signaling pathways is often mediated by upregulation of alternative or compensatory signaling pathways. ${ }^{32-34}$ Our findings suggest that increased glycolytic rates may also have an important role in the development of resistance, which supports the previously published data demonstrating that increased glycolysis contributes to lapatinib, trastuzumab and paclitaxel resistance in tumor cells. ${ }^{35-37}$

Akt has a key role in multiple cellular processes such as cell proliferation and survival as well as energy metabolism and chemoresistance ${ }^{38-40}$ Akt was previously reported to induce a dose-dependent stimulation of glycolysis that correlates with a more aggressive malignancy in vivo. ${ }^{41}$ Treatment with axitinib resulted in increased pAkt as early as $30 \mathrm{~min}$ and was still sustained after $24 \mathrm{~h}$ (Figures $5 \mathrm{a}$ and $\mathrm{b}$ ). By blocking pAkt with a PI3K inhibitor, we reversed the Glut-1 translocation and restored sensitivity to axitinib treatment. By combining axitinib with an Akt inhibitor (MK-2206) to disrupt Akt-mediated glycolytic activation in parental mouse PDAC cells, we observed a decrease in cell viability beyond that conferred by either therapy alone. Furthermore, in axitinib-resistant cell lines an Akt inhibitor (MK-2206) reversed the translocation of Glut-1 to cytosolic pools resulting in a 'normalization' of glucose metabolism and restored sensitivity to axitinib treatment. Previously, vandetanib, an epidermal growth factor receptor (EGFR) and VEGFR inhibitor also increased pAkt in salivary gland tumor cells resulting in the development of resistance. ${ }^{42}$ Increased pAkt could be mediated by activation of other receptor tyrosine kinase signaling pathways, in response to inhibition of the pathway the TKI is targeting. Additionally, off target effects may cause as yet unknown effects resulting from interactions with other proteins.

Interestingly, we also observed a dose-dependent increase in oxygen consumption in parental PDAC cells treated with axitinib and a 2- to 3-fold higher oxygen consumption rate is maintained in the axitinib-resistant cell clones compared with the parental PDAC control (data not shown). This suggests that increased oxidative phosphorylation rates as well as glycolysis may have an important role in the development of resistance. ${ }^{43-45}$ Although pAKT-mediated Glut-1 transport to the membrane results in increased glycolysis ${ }^{41}$ how this may lead to increased mitochondrial respiration is not well understand. Activated Akt also increases HKII association with the mitochondrial membrane, ${ }^{46}$ which prevents initiation of apoptosis ${ }^{47,48}$ and increases oxidative respiration. ${ }^{49}$ It is therefore possible that this may be the cause of the increased oxidative phosphorylation we observe in response to axitinib treatment.

In the clinical setting, the major pathway in which axitinib mediates its anti-tumor effects is via inhibition of VEGF signaling/angiogenesis and remodeling of the 
a
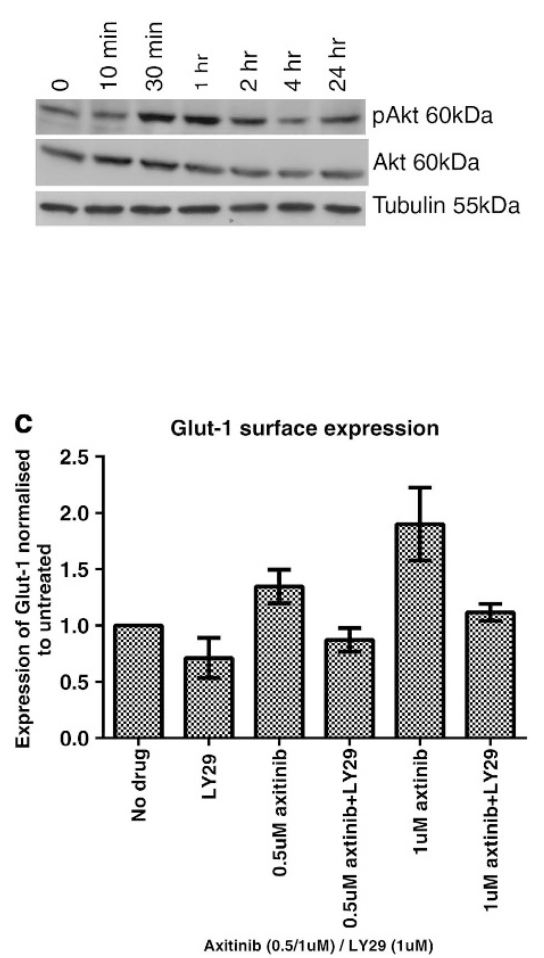

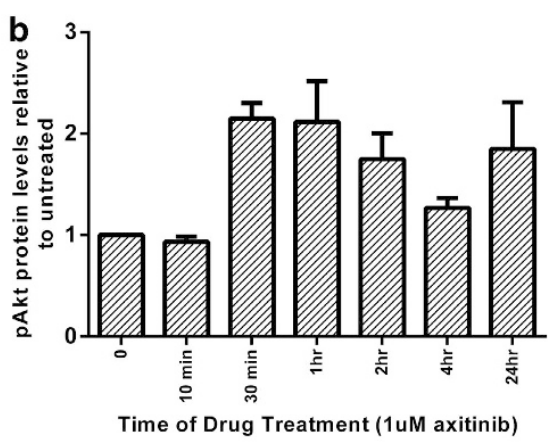

d

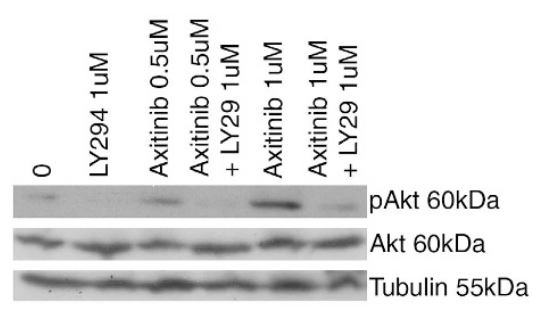

Figure 5 Increased Glut-1 translocation to the membrane is mediated via axitinib-induced activation of Akt. (a) Mouse PDAC cells were treated with $1 \mu$ M axitinib for the indicated times and pAkt and total Akt were analyzed by immunoblotting. (b) Graphical representation of (a) ratio of pAKT protein levels, normalized to total AKT levels and relative to untreated. Data represent mean and S.D. and experiments were repeated at least three times. (c) Glut-1 surface expression was quantified by flow cytometry using Glut-1 conjugated to PE. Cells were treated with either $1 \mu \mathrm{M}$ LY294002 or axitinib at the indicated concentrations and in combination for $24 \mathrm{~h}$. (d) pAKT and total AKT were assessed by western blotting in cells treated with either $1 \mu \mathrm{M} \mathrm{LY} 294002$ or axitinib at the indicated concentrations and in combination for $24 \mathrm{~h}$

host/tumor environment. ${ }^{3,50}$ It has also been demonstrated in vitro that axitinib downregulates the expression of the transporters ABCG2 and ATP7A that are associated with drug resistance in pancreatic cancer cells alone and in combination with $\mathrm{SN}-38$, an active metabolite of irinotecan. ${ }^{24}$ Further in vivo experiments are warranted to assess the resistance pathway found in this study. Furthermore, in the pancreatic cell lines tested, we saw no expression of axitinib receptor targets (VEGFR-2, PDGFR and C-kit) at the protein level, suggesting that axitinib may be acting through as of yet unidentified targets in pancreatic cancer cells, which is in line with the well-recognized target promiscuity of receptor tyrosine kinase inhibitors (RTKIs) such as axitinib.

In summary, we identified an upregulation of the pAktglycolytic axis soon after axitinib treatment as well as in cells surviving axitinib treatment, which was maintained in axitinibresistant cell lines. We demonstrated that treatment of axitinib-resistant PDAC cells with the Akt-specific inhibitor Mk-2206 restored sensitivity to axitinib. Further in vitro studies demonstrated that the Akt inhibitor/axitinib combination provide a significant improvement in anti-tumor effects and provide a rational to test this combination further using in vivo models. Increased activation of Akt may be upregulating Glut-1 transporter expression via increased activity of mammalian target of rapamycin complex 1 (mTORC1), ${ }^{51}$ indicating a possible strategy of combinational axitinib and mTOR inhibitors in pancreatic cancer treatment. As both signaling and metabolic changes are associated with axitinib resistance in pancreatic cancer, targeting the compensatory metabolic networks may be an effective strategy against acquired resistance to targeted therapies.

\section{Materials and Methods}

Reagents. Axitinib was purchased from LC Laboratories (Woburn, MA, USA) and LY2949002 from CayMan Chemical (Ann Arbor, MI, USA), 2-deoxy-D-glucose (2DG) grade III was purchased from Sigma-Aldrich (St. Louis, MO, USA) and Akt-specific inhibitor (MK-2206) was purchased from Selleck Chemicals (Houston, TX, USA).

Cell culture. Human pancreatic cell lines Panc1, Miapaca SUIT-2 and BXPC-3 were provided by Dr. Hagemann and Dr. M Yaun at The Bart's Cancer Institute. All human cell lines were authenticated (LGC Standards, Middlesex, UK). Mouse PDAC and PanIN cell lines were obtained from the spontaneous pancreatic cancer mouse model (Kras $\left.{ }^{G 12 D} P d x 1-c r e\right)$, which recapitulates the features of human PDAC. Cell lines were maintained in high-glucose Dulbecco's modified Eagle's medium (DMEM) supplemented with 10\% fetal bovine serum. Glucose-free medium supplemented with $2 \%$ dialyzed fetal bovine serum was used in the in vitro uptake assays.

Generation of axitinib-resistant PDAC cell lines. The axitinibresistant cell lines were generated by exponentially growing mouse PDAC cells in $10 \mathrm{~cm}$ plates and treating them with $500 \mathrm{nM}$ axitinib for $48 \mathrm{~h}$ followed by a recovery period in drug-free media until the cells regained exponential growth. This process was repeated for eight cycles until the cells demonstrated stable resistance. The resistant cells were serially diluted and each of the resistant cell lines PDAC-R1, PDAC-R2 and PDAC-R3 was cloned separately from a single cell. The resistant cell lines were grown in drug-free media and monitored for resistance every 2 weeks. 
a

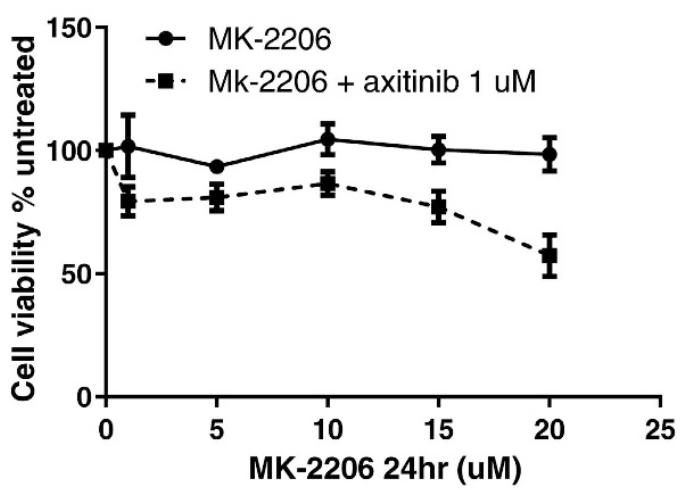

b Parental PDAC cells

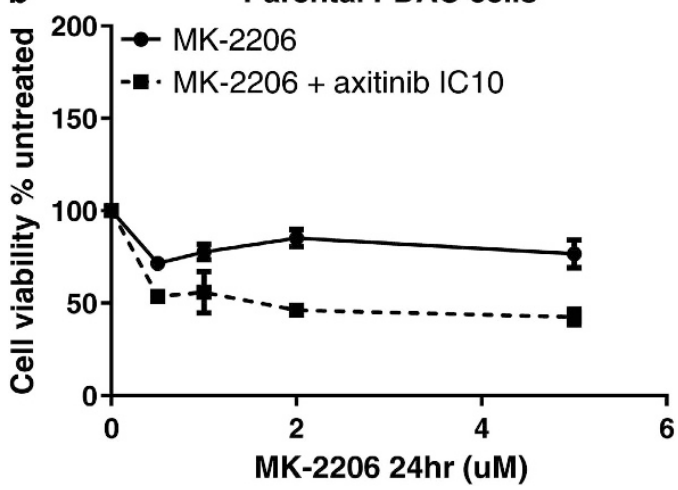

Figure 6 MK-2206 sensitizes cells to axitinib treatment in resistant cell lines and cell viability is reduced upon dual treatment compared with single treatments alone. (a) Axitinib-resistant mouse PDAC cells were treated with increasing concentrations of MK-2206 with and without $1 \mu \mathrm{M}$ axitinib. After $24 \mathrm{~h}$ treatment, cell viability was assessed by Vi-CELL trypan blue counter. Mean \pm S.D. percent viable cells compared with untreated. Experiments were performed in duplicate and repeated three times. (b) Mouse PDAC cells were treated with increasing concentrations of MK-2206 with and without $\mathrm{IC}_{10}$ concentration of axitinib. After $24 \mathrm{~h}$ treatment, cell viability was assessed by Vi-CELL trypan blue counter. Mean \pm S.D. percent viable cells are expressed relative to untreated control. Experiments were performed in triplicate and repeated three times

Cell viability assay. The growth inhibitory effects of axitinib, 2-DG, the PI3K inhibitor LY294002 and the Akt-specific inhibitor MK-2206 were measured using the Vi-CELL trypan blue exclusion counter (Beckman Coulter, Brea, CA, USA). Cells were plated out in 6 -well plates (Corning, Corning, NY, USA) at $1 \times 10^{5}$ cells per well the night before drug treatment. Cells were then incubated with different drug concentrations or combinations as stated for either 24 or $48 \mathrm{~h}$. Viable cell numbers from triplicate wells were then determined using the Vi-CELL counter (Beckman coulter). $I \mathrm{I}_{50}$ values were obtained using the Prism Software (GraphPad, La Jolla, CA, USA) and plotted in dose response curves.

Protein expression. Protein was isolated from cells using lysis buffer $(20 \mathrm{mM}$ Tris pH 8, $200 \mathrm{mM} \mathrm{NaCl}, 1 \mu \mathrm{m}$ EDTA pH $80.5 \%$ NP-40 and 10\% glycerol). Lysates were standardized for protein content and $20 \mu \mathrm{g}$ of total protein was separated by $10 \%$ SDS-polyacrylamide gel electrophoresis and transferred onto PVDF membranes using the iBlot system (Invitrogen, Grand Island, NY, USA). The membranes were blocked and primary antibody was diluted $1 / 1000$ in $3 \%$ BSA in TBS containing $0.01 \%$ Tween-20 (TBST) and incubated overnight at $4{ }^{\circ} \mathrm{C}$. The following day, blots were washed in TBST buffer and incubated with goat anti-rabbit horseradish peroxidase-conjugated secondary antibody (Dako, Carpinteria, CA, USA) for $1 \mathrm{~h}$ at room temperature. After washing in TBST buffer (three times, $10 \mathrm{~min}$ each wash) the immunoreactive proteins were visualized using ECL (Amersham Biosciences, Pittsburgh, PA, USA). The antibodies used were Akt (Total) and pAkt (ser473) from Cell Signaling (Danvers, MA, USA), Glut-1 (Millipore, Billerica, MA, USA), MCT-4 and $\beta$-tubulin (Santa Cruz, Santa Cruz, CA, USA).
In vitro tracer uptake assays. Cells were plated at $1 \times 10^{5}$ cells per well the evening before the experiment. The next day cells were incubated with drug for 24 or $48 \mathrm{~h}$. On the day of the assay, the drug-containing media was removed and cells incubated in glucose free-media supplemented with $2 \%$ dialyzed fetal bovine serum for $1 \mathrm{~h}$. [C-14]DG was then added at $1 \mu \mathrm{Ci} / \mathrm{ml}$ to each well and incubated for a further $1 \mathrm{~h}$. Cells were then washed twice in ice-cold PBS, and harvested with trypsin. The cell lysates were collected and added to tubes containing $2 \mathrm{ml}$ of scintillation fluid and the cell associated [C-14] radioactivity was measured using the $\beta$ scintillation counter (LKB Instruments, Mt Waverley, VIC, Australia). Radioactivity uptake is expressed as nmoles uptake of tracer per 10000 live cells and expressed relative to untreated controls. All experiments were performed in triplicate and repeated three times.

Cell-cycle analysis. Cells were plated out at $0.5 \times 10^{5}$ per well the evening before the experiment. The cells were then serum starved for $24 \mathrm{~h}$ before axitinib treatment at the indicated concentrations for $24 \mathrm{~h}$. The cells were then harvested by trypsinization, washed twice in cold PBS, fixed with ice-cold 70\% ethanol and incubated at $4^{\circ} \mathrm{C}$ overnight. Cells were then washed with PBS and incubated with $50 \mu \mathrm{g} / \mathrm{ml}$ propidium iodide (PI) and $100 \mu \mathrm{g} / \mathrm{ml}$ ribonuclease $\mathrm{A}$ for $30 \mathrm{~min}$ at $37^{\circ} \mathrm{C}$. Cells were analyzed on the Fortessa flow cytometer (BD, San Jose, CA, USA) and results were analyzed using the FlowJo Software (Ashland, OR, USA). Experiments were done in duplicate and repeated at least three times.

FACS analysis for Glut-1 expression. Cells were plated out at $1 \times 10^{5}$ cells per well the evening before the experiment. The next day cells were incubated with the indicated concentrations of axitinib and/or LY2949002 for $24 \mathrm{~h}$ and harvested using versene (Invitrogen). Cells were then washed in ice-cold FACS buffer (PBS $+2 \%$ FCS) and resuspended in $50 \mu$ l of PBS. In all, $5 \mu$ l of PE labeled Glut-1 (R\&D Systems, Minneapolis, MN, USA) was added and the cells were incubated on ice for $30 \mathrm{~min}$. Cells were then washed twice in FACS buffer and resuspended in $400 \mu \mathrm{l}$ of FACS buffer containing $0.5 \mu \mathrm{g} / \mathrm{ml}$ of 4,6-diamidino-2phenylindole (DAPI). Glut-1 surface expression in the live cell population was analyzed on the Fortessa flow cytometer $(\mathrm{BD})$ and the results were analyzed using FlowJo software. Experiments were performed in duplicate and repeated at least three times.

Immunofluorescence microscopy. Cells were seeded and incubated $\mathrm{O} / \mathrm{N}$ on coverslips in 24-well plates. The following day cells were treated with axitinib for $24 \mathrm{~h}$. Cells were then washed three times in TBS and fixed in $3.7 \%$ formaldehyde for $20 \mathrm{~min}$. After washing in TBS cells were permeabilized in TBST (TBS $+0.1 \%$ Triton X-100) for $10 \mathrm{~min}$, followed by washes in TBS. The coverslips were then blocked in TBS containing $10 \%$ goat serum for 20 min followed by incubation in Glut-1 antibody in TBST $+1 \%$ serum. Cells were then washed three times in TBS and incubated in anti-rabbit labeled secondary followed by five washes in TBS. The cells were then mounted in anti-fade medium containing DAPI (Invitrogen). Images were obtained using the Zeiss LSM 510 (Jena, Germany).

Reverse transcriptase-PCR. Relative expression ratio of Glut- 1 was measured using qRT-PCR. Total RNA was extracted from PDAC cells treated with axitinib $(0-1 \mu \mathrm{m})$ using the RNeasy kit (Qiagen, Valencia, CA, USA). Total RNA $(2 \mu \mathrm{g})$ was reverse transcribed to cDNA (High Capacity cDNA Reverse Transcription Kit, Applied Biosystems, Grand Island, NY, USA) using primers for the target gene Glut-1 (TaqMan Gene Expression Assays ID: Mm00441473_m1) and 18S internal control (Taqman Control ID: Mm03928990_g1) from Applied Biosystems. PCR amplification was carried out using the ABI 7500 qRT PCR system (Applied Biosystems), under the following conditions: $50^{\circ} \mathrm{C}$ for $2 \mathrm{~min}$, $95^{\circ} \mathrm{C}$ for $10 \mathrm{~min}$ and 40 cycles of $95^{\circ} \mathrm{C} 15 \mathrm{~s}$ and $60^{\circ} \mathrm{C}$ for $1 \mathrm{~min}$. Experiments were carried out in triplicate and repeated three times.

ECAR analysis. The XF24 extracellular flux analyzer (Seahorse Biosciences) is a fully integrated 24-well instrument that measures in real time the uptake and release of metabolic end products. Each XF24 assay well contains a disposable sensor cartridge, embedded with 24 pairs of fluorescent biosensors (oxygen and $\mathrm{pH}$ ), coupled to fiber-optic waveguides. This technology was used to measure ECAR expressed in $\mathrm{mpH} / \mathrm{min}$ in control, treated and axitinib-resistant cell lines. 
Statistical analysis. Mean values and S.D. were calculated using Microsoft Excel or Prism software. The analysis of variance (ANOVA) was used to identify statistical differences between experimental factors. $P<0.05$ was considered as statistically significant.

\section{Conflict of Interest}

The authors declare no conflict of interest.

Acknowledgements. We would like to thank Dr. Guglielmo Rosignoli from the Flow Cytometry core Facility for support and training and Alyssa Savadelis for helping in additional studies for revising the manuscript. This work was supported by the Bart's and the London Trust (BLT) Charitable Foundation, London, UK; Case Western Reserve University and Ohio Third Frontier Fund.

1. Moore PS, Beghelli S, Zamboni G, Scarpa A. Genetic abnormalities in pancreatic cancer Mol Cancer. 2003; 2: 7

2. Zhang J, Yang PL, Gray NS. Targeting cancer with small molecule kinase inhibitors. Nat Rev Cancer 2009; 9: 28-39.

3. Hu-Lowe DD, Zou HY, Grazzini ML, Hallin ME, Wickman GR, Amundson K et al. Nonclinical antiangiogenesis and antitumor activities of axitinib (AG-013736), an oral, potent, and selective inhibitor of vascular endothelial growth factor receptor tyrosine kinases 1, 2, 3. Clin Cancer Res 2008; 14: 7272-7283.

4. Nio $\mathrm{Y}$, Omori H, Toga T, Hashimoto K, Itakura M, Koike M et al. Immunohistochemical expression of receptor-tyrosine kinase c-kit protein in invasive ductal carcinoma of the pancreas. Anticancer Drugs 2003; 14: 313-319.

5. Ebert M, Yokoyama M, Friess H, Kobrin MS, Buchler MW, Korc M. Induction of platelet-derived growth factor $\mathrm{A}$ and $\mathrm{B}$ chains and over-expression of their receptors in human pancreatic cancer. Int J Cancer 1995; 62: 529-535.

6. Itakura J, Ishiwata $\mathrm{T}$, Friess $\mathrm{H}$, Fujii $\mathrm{H}$, Matsumoto $\mathrm{Y}$, Buchler $\mathrm{MW}$ et al. Enhanced expression of vascular endothelial growth factor in human pancreatic cance correlates with local disease progression. Clin Cancer Res 1997; 3: 1309-1316.

7. Rixe O, Bukowski RM, Michaelson MD, Wilding G, Hudes GR, Bolte $O$ et al. Axitinib treatment in patients with cytokine-refractory metastatic renal-cell cancer: a phase II study. Lancet Oncol 2007; 8: 975-984.

8. Rini BI, Wilding G, Hudes G, Stadler WM, Kim S, Tarazi J et al. Phase II study of axitinib in sorafenib-refractory metastatic renal cell carcinoma. J Clin Oncol 2009; 27: 4462-4468

9. Spano JP, Chodkiewicz C, Maurel J, Wong R, Wasan H, Barone C et al. Efficacy of gemcitabine plus axitinib compared with gemcitabine alone in patients with advanced pancreatic cancer: an open-label randomised phase II study. Lancet 2008; 371 2101-2108

10. Cohen EE, Rosen LS, Vokes EE, Kies MS, Forastiere AA, Worden FP et al. Axitinib is an active treatment for all histologic subtypes of advanced thyroid cancer: results from a phase II study. J Clin Oncol 2008; 26: 4708-4713.

11. Schiller JH, Larson T, Ou SH, Limentani S, Sandler A, Vokes E et al. Efficacy and safety of axitinib in patients with advanced non-small-cell lung cancer: results from a phase II study. $J$ Clin Oncol 2009; 27: 3836-3841.

12. Fruehauf J, Lutzky J, McDermott D, Brown CK, Meric JB, Rosbrook B et al Multicenter, phase II study of axitinib, a selective second-generation inhibitor of vascula endothelial growth factor receptors 1,2 , and 3 , in patients with metastatic melanoma. Clin Cancer Res 2011; 17: 7462-7469.

13. Kindler HL, loka T, Richel DJ, Bennouna J, Letourneau R, Okusaka $T$ et al. Axitinib plus gemcitabine versus placebo plus gemcitabine in patients with advanced pancreatic adenocarcinoma: a double-blind randomised phase 3 study. Lancet Oncol 2011; 12: 256-262.

14. Sierra JR, Cepero V, Giordano S. Molecular mechanisms of acquired resistance to tyrosine kinase targeted therapy. Mol Cancer 2010; 9: 75

15. Butler EB, Zhao Y, Munoz-Pinedo C, Lu J, Tan M. Stalling the engine of resistance: targeting cancer metabolism to overcome therapeutic resistance. Cancer Res 2013; 73 2709-2717.

16. Aiura K, Takahashi S, Matsui J, Ueda M, Kitagawa Y. Beneficial effects of 5-Fluorouracil and heparin-based portal infusion chemotherapy combined with mitomycin $C$ and cisplatin after curative resection of pancreatic cancer. Pancreatology 2010; 10 : 250-258.

17. Takahashi R, Hirata H, Tachibana I, Shimosegawa E, Inoue A, Nagatomo I et al. Early [18F]fluorodeoxyglucose positron emission tomography at two days of gefitinib treatment predicts clinical outcome in patients with adenocarcinoma of the lung Clin Cancer Res 2012; 18: 220-228.

18. Su H, Bodenstein C, Dumont RA, Seimbille $Y$, Dubinett $S$, Phelps ME et al Monitoring tumor glucose utilization by positron emission tomography for the prediction of treatment response to epidermal growth factor receptor kinase inhibitors. Clin Cancer Res 2006; 12: 5659-5667.
19. Jadvar H, Alavi A, Gambhir SS. 18F-FDG uptake in lung, breast, and colon cancers: molecular biology correlates and disease characterization. J Nucl Med 2009; 50: 1820-1827.

20. Mileshkin L, Hicks RJ, Hughes BG, Mitchell PL, Charu V, Gitlitz BJ et al. Changes in 18Ffluorodeoxyglucose and 18F-fluorodeoxythymidine positron emission tomography imaging in patients with non-small cell lung cancer treated with erlotinib. Clin Cancer Res 2011; 17: 3304-3315.

21. Rockall A, Munari A, Avril N. New ways of assessing ovarian cancer response: metabolic imaging and beyond. Cancer Imaging 2012; 12: 310-314.

22. Schwarz-Dose J, Untch M, Tiling R, Sassen S, Mahner S, Kahlert S et al. Monitoring primary systemic therapy of large and locally advanced breast cancer by using sequential positron emission tomography imaging with [18F]fluorodeoxyglucose. J Clin Oncol 2009; 27: $535-541$

23. Rossler J, Monnet Y, Farace F, Opolon P, Daudigeos-Dubus E, Bourredjem A et al. The selective VEGFR1-3 inhibitor axitinib (AG-013736) shows antitumor activity in human neuroblastoma xenografts. Int J Cancer 2011; 128: 2748-2758.

24. Canu B, Fioravanti A, Orlandi $\mathrm{P}$, Di Desidero $\mathrm{T}$, Ali G, Fontanini $\mathrm{G}$ et al. Irinotecan synergistically enhances the antiproliferative and proapoptotic effects of axitinib in vitro and improves its anticancer activity in vivo. Neoplasia 2011; 13: 217-229.

25. Haberkorn U, Bellemann ME, Altmann A, Gerlach L, Morr I, Oberdorfer F et al. PET 2-fluoro-2-deoxyglucose uptake in rat prostate adenocarcinoma during chemotherapy with gemcitabine. J Nucl Med 1997; 38: 1215-1221.

26. Pinheiro C, Longatto-Filho A, Azevedo-Silva J, Casal M, Schmitt FC, Baltazar F. Role of monocarboxylate transporters in human cancers: state of the art. J Bioenerg Biomembr 2012; 44: 127-139.

27. Schneiderhan W, Scheler M, Holzmann KH, Marx M, Gschwend JE, Bucholz M et al. CD147 silencing inhibits lactate transport and reduces malignant potential of pancreatic cancer cells in in vivo and in vitro models. Gut 2009; 58: 1391-1398.

28. de Wit M, Jimenez CR, Carvalho B, Belien JA, Delis-van Diemen PM, Mongera S et al. Cell surface proteomics identifies glucose transporter type 1 and prion protein as candidate biomarkers for colorectal adenoma-to-carcinoma progression. Gut 2012; 61: 855-864.

29. Macheda ML, Rogers S, Best JD. Molecular and cellular regulation of glucose transporter (GLUT) proteins in cancer. J Cell Physiol 2005; 202: 654-662.

30. Kalir T, Wang BY, Goldfischer M, Haber RS, Reder I, Demopoulos R et al. Immunohistochemical staining of GLUT1 in benign, borderline, and malignant ovarian epithelia. Cancer 2002; 94: 1078-1082.

31. Tarn C, Skorobogatko YV, Taguchi T, Eisenberg B, von Mehren M, Godwin AK. Therapeutic effect of imatinib in gastrointestinal stromal tumors: AKT signaling dependent and independent mechanisms. Cancer Res 2006; 66: 5477-5486.

32. Kobayashi S, Boggon TJ, Dayaram T, Janne PA, Kocher O, Meyerson M et al. EGFR mutation and resistance of non-small-cell lung cancer to gefitinib. New Engl J Med 2005; 352: 786-792.

33. Engelman JA, Zejnullahu K, Mitsudomi T, Song Y, Hyland C, Park JO et al. MET amplification leads to gefitinib resistance in lung cancer by activating ERBB3 signaling. Science 2007; 316: 1039-1043.

34. Sharma SV, Lee DY, Li B, Quinlan MP, Takahashi F, Maheswaran S et al. A chromatinmediated reversible drug-tolerant state in cancer cell subpopulations. Cell 2010; 141:69-80.

35. Komurov K, Tseng JT, Muller M, Seviour EG, Moss TJ, Yang L et al. The glucosedeprivation network counteracts lapatinib-induced toxicity in resistant ErbB2-positive breast cancer cells. Mol Syst Biol 2012; 8: 596.

36. Zhao Y, Liu H, Liu Z, Ding Y, Ledoux SP, Wilson GL et al. Overcoming trastuzumab resistance in breast cancer by targeting dysregulated glucose metabolism. Cancer Res 2011; 71: 4585-4597

37. Zhou M, Zhao Y, Ding Y, Liu H, Liu Z, Fodstad O et al. Warburg effect in chemosensitivity: targeting lactate dehydrogenase-A re-sensitizes taxol-resistant cancer cells to taxol. $\mathrm{Mol}$ Cancer 2010; 9: 33

38. Kennedy SG, Wagner AJ, Conzen SD, Jordan J, Bellacosa A, Tsichlis PN et al. The PI 3-kinase/Akt signaling pathway delivers an anti-apoptotic signal. Genes Dev 1997; 11: $701-713$.

39. Hahn-Windgassen A, Nogueira V, Chen CC, Skeen JE, Sonenberg N, Hay N. Akt activates the mammalian target of rapamycin by regulating cellular ATP level and AMPK activity. J Biol Chem 2005; 280: 32081-32089.

40. Huang WC, Hung MC. Induction of Akt activity by chemotherapy confers acquired resistance. J Formos Med Assoc 2009; 108: 180-194.

41. Elstrom RL, Bauer DE, Buzzai M, Karnauskas R, Harris MH, Plas DR et al. Akt stimulates aerobic glycolysis in cancer cells. Cancer Res 2004; 64: 3892-3899.

42. Fujishiro Y, Tonogi M, Ochiai H, Matsuzaka K, Yamane GY, Azuma T. The receptor tyrosine kinase inhibitor vandetanib activates Akt and increases side population in a salivary gland tumor cell line (A253). Int J Oncol 2012; 41: 362-368.

43. Baffy G, Derdak Z, Robson SC. Mitochondrial recoupling: a novel therapeutic strategy for cancer? Br J Cancer 2011; 105: 469-474.

44. Palorini R, Simonetto T, Cirulli C, Chiaradonna F. Mitochondrial complex I inhibitors and forced oxidative phosphorylation synergize in inducing cancer cell death. Int J Cell Biol 2013; 2013: 243876 .

45. Suganuma K, Miwa H, Imai N, Shikami M, Gotou M, Goto M et al. Energy metabolism of leukemia cells: glycolysis versus oxidative phosphorylation. Leuk Lymphoma 2010; 51 2112-2119. 
46. Majewski N, Nogueira V, Bhaskar P, Coy PE, Skeen JE, Gottlob K et al. Hexokinase-mitochondria interaction mediated by Akt is required to inhibit apoptosis in the presence or absence of Bax and Bak. Mol Cell 2004; 16: 819-830.

47. Pastorino JG, Shulga N, Hoek JB. Mitochondrial binding of hexokinase II inhibits Bax-induced cytochrome c release and apoptosis. J Biol Chem 2002; 277: 7610-7618.

48. Gottlob K, Majewski N, Kennedy S, Kandel E, Robey RB, Hay N. Inhibition of early apoptotic events by Akt/PKB is dependent on the first committed step of glycolysis and mitochondrial hexokinase. Genes Dev 2001; 15: 1406-1418.

49. Mathupala SP, Ko YH, Pedersen PL, Hexokinase II. cancer's double-edged sword acting as both facilitator and gatekeeper of malignancy when bound to mitochondria. Oncogene 2006; 25: 4777-4786.

50. Wilmes LJ, Pallavicini MG, Fleming LM, Gibbs J, Wang D, Li KL et al. AG-013736, a novel inhibitor of VEGF receptor tyrosine kinases, inhibits breast cancer growth and decreases vascular permeability as detected by dynamic contrast-enhanced magnetic resonance imaging. Magn Reson Imaging 2007; 25: 319-327.
51. Brugarolas JB, Vazquez F, Reddy A, Sellers WR, Kaelin WG Jr. TSC2 regulates VEGF through mTOR-dependent and -independent pathways. Cancer Cell 2003; 4: 147-158.

(c) (i)(-)(2) Cell Death and Disease is an open-access journal published by Nature Publishing Group. This work is licensed under a Creative Commons Attribution-NonCommercialShareAlike 3.0 Unported License. The images or other third party material in this article are included in the article's Creative Commons license, unless indicated otherwise in the credit line; if the material is not included under the Creative Commons license, users will need to obtain permission from the license holder to reproduce the material. To view a copy of this license, visit http://creativecommons.org/licenses/ by-nc-sa/3.0/ 